\title{
Synthesizing the Artistic Effects of Ink Painting
}

\author{
Ching-tsorng Tsai ${ }^{1}$, Chishyan Liaw ${ }^{1}$, Cherng-yue Huang ${ }^{1}$, and Jiann-Shu Lee ${ }^{2}$ \\ ${ }^{1}$ Department of Computer Science and Information Engineering, Tunghai University, \\ 181 Sec. 3, Taichung-Kung Rd., Taichung 407, Taiwan \\ \{cttsai, liaw\}@mail.thu.edu.tw \\ ${ }^{2}$ Department of Information and Learning Technology, National University of Tainan, \\ 33 Sec. 2, Shu-Lin St., Tainan 412, Taiwan
}

\begin{abstract}
A novel method that is able to simulate artistic effects of ink-refusal and stroke-trace-reservation in ink paintings is developed. The main ingredients of ink are water, carbon particles, as well as glue. However, glue is not taken into account in other researches, although it plays an important role in ink diffusion. In our ink-diffusion model, we consider the number of fibers and the quantity of glue as parameters of the structure of paper. We simulate the physical interaction among water, carbon particles, glue, and fiber mesh of paper. The realistic renderings created from our models have demonstrated that our models are successful, and are able to imitate the special artistic effects of ink painting.
\end{abstract}

\section{Introduction}

Ink painting has been in the Orient for thousands of years. It is a kind of remarkable non-photorealistic rendering; with a few strokes in gray tones, it is still able to depict its implicit spirit and meanings. The ancient ink painting techniques can be simulated and imitated by using computer software. Many researchers have demonstrated their models and results, but none of them have found a simple and yet effective model to achieve the special effects of ink-refusal and stroke-trace-reservation, that appear in ink paintings. Ink painting involves a complex interaction of its art mediums, and is very difficult to be imitated by using a simple model. Strassmann [1] applied texturemapping techniques to simulate the diffusion of ink painting. Some $[2,3,4]$ tried to render the silhouette with stylized strokes, while others, like Small [5] and Curtis [6] thought the ink was similar to the material used in watercolor and applied the watercolor diffusion model on ink diffusion situations. However, it takes a lot of effort to calculate, and is difficult to effective diffusion.

Guo [7] presented the diffusion speed function and classified the carbon particles in ink and the fiber density of paper for four levels. Kunii [8] constructed a multidimensional diffusion model to describe the phenomena of ink diffusion. Water spreads in the paper due to microscopic effects, but the movement of carbon particles, which are much bigger than water molecules, is based on Brownian motion. Lee [9] observed that the displacement of diffused ink front in still water agreed with the theory of diffusion proposed by Kunii. Based on Kunii's diffusion equations, Wang 
[10] also took the structure of paper and gravity into account. Huang [11] divided the paper into many 2D Papels that have their own base according to the kinds of fiber. By adding the number of fibers to the base and multiplying a random number, one can calculate the absorbency of paper. Zhang [12] presented a diffusion model based on a 2D cellular automaton, a 2D grid array of tanks linked with their neighbors by pipes, computational model. He developed models of the transfer and diffusion between cells. Guo [13] defined each point of the fiber mesh as fiber structure data and capillary structure data, which includes the number of fibers and capillary tubes connected to eight neighboring points. Liquid ink flows along the capillary tubes between interlacing fibers from one point to others. All of the above researches focus on the interaction between water and carbon particles in ink, and fiber distributions in paper. However, another important factor, glue, to diffusion was neglected. The ingredients of ink are water, carbon particles, and glue. The rate of carbon to glue varies from 100:60 to 100:120, so the glue in the ink must play an important role of ink diffusion on absorbent painting paper.

This paper discusses the diffusion rendering of ink painting and focuses on synthesizing artistic effects of ink-refusal and stroke-trace-reservation, which make the ink paintings remarkable, unpredictable, and implicitly beautiful. We derive the diffusion model from the physical interaction between ink and paper. Unlike those previous researches, glue is just as important as the water, carbon, and paper, which are all being taken into account in this paper.

\section{The Characteristics of Ink Painting}

Ink painting mainly uses the ink permeation on special absorbent paper as well as skill with some strokes to express the painter's style and imagination. The ink is composed of water, carbon particles, and glue. Generally, artists use water to control the intensity of color and diffusion. More water in the ink means more diffusion and less intensity in color. Water also serves as the carrier of carbon particles. After the ink carried in the brush touches absorbent paper, carbon particles move along with water into the fiber mesh of the paper. Those particles will be stuck on the fiber mesh with glue after the water dries out; also, higher concentration of glue in ink reduces the diffusion zone of brush strokes.

There are several kinds of absorbent paper used in ink painting. All of them are thin, textured and with high absorbency, which allow the liquid ink to flow and diffuse easily. The paper, like fiber mesh, consists of lots of fibers in random positions and orientations, with some space among them. The phenomenon of ink diffusion on paper is dependent on the surface, thickness, density, impurity, kind of fiber, absorbency, and so on.

After ink is applied to the surface of paper, the space among the fibers serves as capillaries to carry ink away from its initial zone. Interactions among the ingredient molecules of the ink can also cause diffusion. When ink seeps into the fiber mesh, the carbon particles flow with water. The border of the stroke becomes blurry and feathery beauty. The diffusion stops when the amount of water left less than the space among ambient fibers. 
The amount of ink permeated into paper is also relates to the order of stroke in the overlap area. It is difficult for the ink of next stroke to seep into the fiber mesh if it has been filled up on the first stroke. That means the stroke cannot be covered by another stroke. If there are overlapping areas between two strokes, the second stroke doesn't usually darken that same area. That is because ink has permeated and diffused into paper mesh on the former stroke. The structure of its fiber mesh has been changed, filled up with carbon particles and glue after water dried out, and therefore, the quantity of more ink that can seep into the former stroke area is limited. This is the effect of ink-refusal. Fig. 1 shows the effects of ink-refusal and stroke-trace reservation in ink-painting.

When ink permeates and diffuses in the fiber mesh on the former stroke, the water and glue molecules move faster and farther than carbon particles. Glue sticks on the fiber mesh near edge of diffusion area after water dries. There will not have more space for the ink of later stroke. Consequently, it cannot accept more ink on the border of the former stroke where later stroke applied, so a lighter border appears. This is the effect of stroke-trace-reservation. The white border will be bigger if there is enough time for the glue of the former stroke to dry. In ink painting, the first stroke looks like floating on the later overlapped ones.

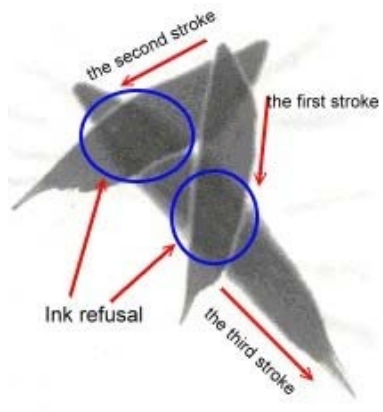

(a)

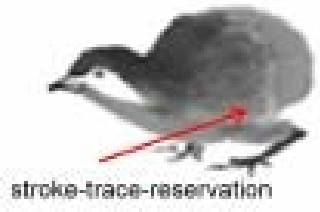

(b)

Fig. 1. The effects of ink-refusal and stroke-trace reservation in ink-painting;(a) bamboo leaves and (b) a chicken

\section{The Proposed Diffusion Model}

In this paper, we describes that the absorbent paper is similar to grid array of fiber tanks linked with their neighbors by pipes. In our model, glue is as important as water and carbon particles. The glue affect the structure of absorbent paper after water evaporates. The interactions among fiber, water, carbon particles, and glue are modeled and all the special effects of ink-refusal and stroke-trace-reservation are well synthesized. 


\subsection{The Structures of Absorbent Paper and Ink}

The absorbent paper consists of lots of fibers in random positions and directions. It is divided into many cells, and each cell relates to its eight neighbor cells. The paper cell, the basic element of paper, is called Papel, which corresponds to a pixel in rendering. $\mathrm{Bi}$ is the base capacity of Papel $\mathrm{i}$ and $\mathrm{Ci}$ represents the capacity of water contained in Papel i. Gi is the quantity of glue and it is set to 0 before ink starts to permeate. Wi represents the quantity of water permeated into Papel i. There is a tube connected between Papel i and its neighbor Papel k. $T H{ }_{k}^{i}$ stands for the minimum quantity of water molecules needed in order to diffuse from Papel i to Papel k, where $T H \underset{k}{i}=B_{i}+C_{i}+G_{i}$.

It is important to construct a data structure of the absorbent paper that is based on its physical properties since different structures generate different diffusion textures. The structure of the paper is defined as:

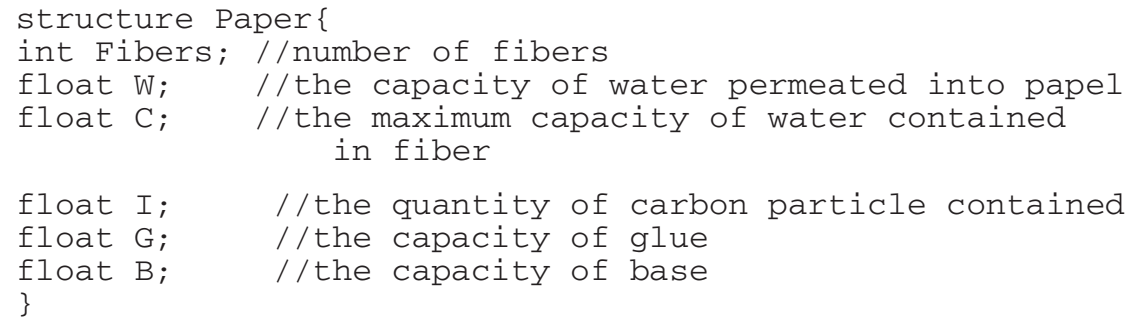

Fibers are generated by Bezier function and distributed in random orientation. The parameter Fibers is added by one if a fiber passed. The capacity of base B and the maximum capacity of water contained in fiber $\mathrm{C}$ can be estimated. In The parameter $\mathrm{H}$ represents for the thickness of the paper. The thickness of the paper and number of fibers provide space for water molecules. The thicker and more fibers, the more ink and water can be contained in Papel and therefore, diffusion zone is less for the same quantity of ink.

Besides, $\mathrm{Bi}$ and $\mathrm{Ci}$ are the quantity of base of impurity such as $\mathrm{CaCo} 3$ and the quantity of water contained in fiber, respectively. They are not identical in different Papels because the fibers are distributed randomly. When a fiber passes Papel i, $\Delta \mathrm{B}$ is subtracted from $\mathrm{Bi}$. Meanwhile, $\mathrm{Ci}$, the capacity of water can be held by fiber, increases the quantity of $\Delta \mathrm{C}$. Therefore,

$$
\left(B_{i}, C_{i}\right):=\left(B_{i}-\Delta B, C_{i}+\Delta C\right) \text {. }
$$

Finally, the minimum quantity of water molecules required in order to overflow on pipe can be figured out.

The quantity of ink seeped into paper when the bristles of the brush touch the surface mainly relates to the speed of the brush movement, the quantity of ink in the brush, and concentration of ink. According to this, we define the structure of ink as 


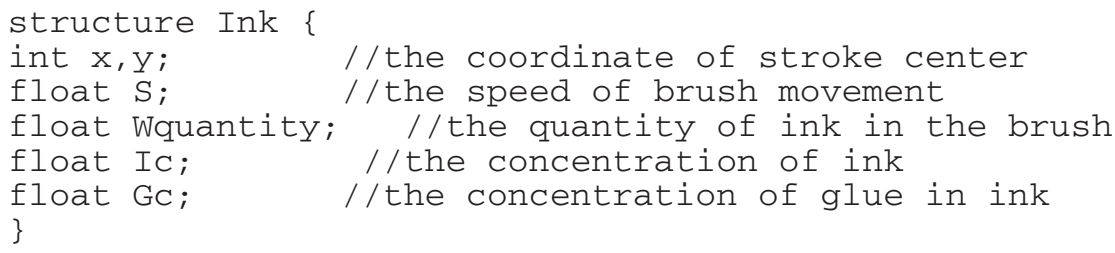

\subsection{Ink Diffusion}

Fig. 2 depicts the process of drying after ink seeped into Papel. The descending of carbon particles and glue change the space among fibers when water evaporates. Therefore, it affects the ink permeation of next stroke on that stroke trace. Fig. 2a shows that there is only base capacity Bi and no any carbon particles and glue in Papel i. Fig. $2 \mathrm{~b}$ represents the condition after first stroke. Wadd, the quantity of ink added, is more than $T H_{k}^{i}$ and it begins to diffuse to neighbor Papels. Fig. 2c shows the situation after the first stroke diffused and dried. Glue, Gi(1), diffused along with water and later clogs the space among fibers. Thus, $\mathrm{Ci}$, the capacity of water can be held by fiber, decreases by $\Delta \mathrm{C}$. Consequently, $T H_{k}^{i}$, the minimum quantity of water molecules needed in order to diffuse from Papel i to Papel k, changes. Fig. 2d represents when the second stroke is added. Obviously, Wadd, the quantity of ink can be added, decreases. This is the cause of the ink-refusal effect. Fig. 2e shows that the solidified glue, Gi(2), clogs more space after the second stroke dry. Fig. 2f shows that the third stroke is added; the capacity of the Papel, $\mathrm{Ci}(3)$, decreases more and the quantity of ink that can be added, Wadd, is even less.

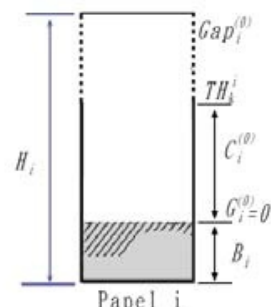

(a)

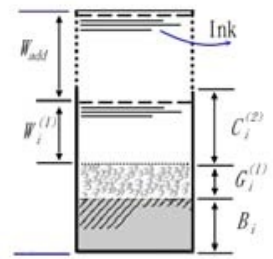

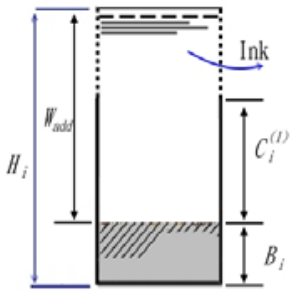

(b)

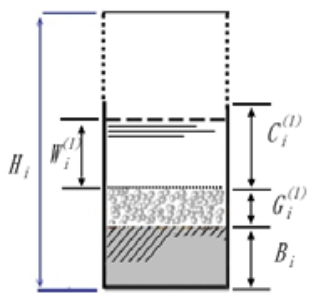

(c)
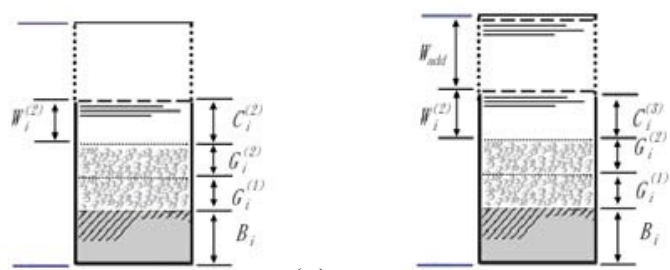

(e)

(d)

Fig. 2. The Papel status; (a)before ink seeps into Papel, (b) when the first stroke is applied (c) after the first stroke diffused and dried, (d) when the second stroke is added,(e) the glue solidified, (f)when the third stroke is applied 
(a)The Diffusion of Water Molecular

Since water molecules are the carriers of carbon particles and glue, carbon particles and glue diffuse only whenever water molecules diffuse. Finally, the carbon particles descend into fibers and are solidified by glue after water molecules evaporate. Fibers, acting like capillaries, carry the ink into the paper mesh when the bristles of the brush touch the surface of the paper. The quantity of ink that seeps into the paper mainly depends on the quantity of ink carried by brush, the speed of brush movement and number of fibers. The ink in the bristles of the brush has plenty of time to seep into the Papel, and diffuse to its neighbor Papels if the brush moves slower. Conversely, there is less or even no ink filled into Papel if the brush moves very fast or the ink runs out in a stroke.The quantity of ink seeped, Wadd, is as following,

$$
\text { Wadd }=(H-B-W)^{*} \text { Wquantity, }
$$

where Wquantity is the quantity of ink carried by the brush. After the ink permeated into the fibers, the water molecules begin to diffuse to its eight neighbor Papels according to the structure of Morre Neighborhood System. The quantity of water permeated into Papel i is

$$
W_{i}:=W_{i}+\sum_{k=0}^{k=7}\left(\Delta W_{i}{ }^{k}-\Delta W_{k}^{i}\right),
$$

where $\Delta W_{i}^{k}$ represents for the quantity of water flows from Papel $\mathrm{k}$ to Papel $\mathrm{i}$ and $\Delta W_{k}^{i}$ is the quantity of water flows from Papel i to Papel k. $\Delta W_{i}^{k}$ and $\Delta W_{k}^{i}$ are defined as

$$
\Delta W_{i}^{k}:=\max \left\{0.0,0.125 \cdot a \cdot \min \left[\left(B_{k}+G_{k}+W_{k}\right)-\left(B_{i}+G_{i}+W_{i}\right),\left(B_{k}+G_{k}+W_{k}\right)-T H_{i}^{k}\right]\right.
$$

and

$$
\Delta W_{k}^{i}:=\max \left\{0.0,0.125 \cdot a \cdot \min \left[\left(B_{i}+G_{i}+W_{i}\right)-\left(B_{k}+G_{k}+W_{k}\right),\left(B_{i}+G_{i}+W_{i}\right)-T H_{k}^{i}\right]\right\},
$$

where $a$ is the coefficient of diffusion, $T H_{i}^{k}$ and $T H_{k}^{i}$ represent the minimum quantity of water required in order to flow from Papel k to Papel i and Papel i to Papel k, respectively. The minimum quantities are calculated as $T H_{i}^{k}:=\max \left\{B_{i}+G_{i}, B_{k}+G_{k}+C_{k}\right\}$ and $T H_{k}^{i}:=\max \left\{B_{k}+G_{k}, B_{i}+G_{i}+C_{i}\right\}$, respectively.

In real ink paintings, the water molecules evaporate gradually. Therefore, some quantity of water is subtracted in each diffusion cycle as the following formula:

$$
W_{i}:=W_{i}-\Delta W .
$$

The diffusion stops when the quantity of water molecules in Papel is less than its minimum value of TH, unless there is more ink added. 
(b)The Diffusion of Carbon Particles

The carbon particles diffuse along with water molecules. The more water diffused, the more carbon particles carried to their neighbor Papels. The diffusion of carbon particles is described in the following equations. Let $\mathrm{I} i$ and $\mathrm{I} k$ denote the quantity of the carbon molecules in Papel i and Papel k, respectively, and Ii is defined as:

$$
I_{i}:=I_{i}+\sum_{k=0}^{k=7}\left(\Delta I_{i}^{k}-\Delta I_{k}^{i}\right)
$$

where $\Delta I_{k}^{i}$ and $\Delta I_{i}^{k}$ are the quantity of carbon particles flow from Papel i to Papel k, and flow from Papel k to Papel i, respectively. $\Delta I_{k}^{i}$ and $\Delta I_{i}^{k}$ are defined as:

$$
\Delta I_{k}^{i}:=\Delta W_{k}^{i}\left(\frac{I_{i}}{W_{i}}\right)
$$

and

$$
\Delta I_{i}^{k}:=\Delta W_{i}^{k}\left(\frac{I_{k}}{W_{k}}\right)
$$

(c)The Diffusion of Glue

The glue in the ink can be dissolved in water. It diffuses, along with water, to its neighbor Papels and then its concentration decreases. The glue sticks on fibers after the water dries. The glue contained in Papel $i$ is

$$
G_{i}:=G_{i}+\sum_{k=0}^{k=7}\left(\Delta G_{i}^{k}-\Delta G_{k}^{i}\right),
$$

where $\Delta G_{i}^{k}$ and $\Delta G_{k}^{i}$ represent the quantity of glue flows from Papel k to Papel i, and the quantity of glue flows from Papel $\mathrm{i}$ to Papel k, respectively. $\Delta G_{i}^{k}$ and $\Delta G_{k}^{i}$ are defined as

$$
\Delta G_{k}^{i}:=\Delta W_{k}^{i}\left(\frac{G_{i}}{W_{i}}\right)
$$

and

$$
\Delta G_{i}^{k}:=\Delta W_{i}^{k}\left(\frac{G_{k}}{W_{k}}\right),
$$

where $\mathrm{G} k$ and $\mathrm{G} i$ are the quantities of glue contained in Papel $\mathrm{k}$ and Papel, respectively.

The glue sticks the fibers and clogs the fiber gaps after water dry out. The ink of the latter stroke will be rejected by the stroke trace because of the effect of inkrefusal. Thus, a white border is formed on the edge of the first stroke and it appears in 
contrast if another stroke overlaid on it. The effect of stroke-trace-reservation makes the first stroke floating on the later strokes. To simulate these phenomena, we have to find the space stuck by glue, $\mathrm{G} i$, in Papel i. The formula is

$$
G_{i}=G_{i}+\alpha \cdot \Delta G_{i} \cdot D_{\text {rate }},
$$

where Drate is the solidification rate of glue, and $0 \leqq$ Drate $\leqq 1$. The larger of Drate is, the more glue solidifies.

\section{Results}

To imitate the permeation of ink and calculate the capacity of water in paper as well as the quantity of carbon particles, we input the stroke and find its boundary. The gray-scale intensity of the area inside the boundary is converted to the amount of carbon particles. The quantities of water and glue are also inputted in order for it to seep into the corresponding Papels. The first bamboo leave is loaded into the model to simulate the diffusion and the effect of ink-refusal. The drying time is approximately forty steps. To simulate the real ink painting, fifteen diffusion steps are taken as the interval between two strokes. Similarly, the second and the third strokes are inputted and simulated. Fig.1a is the image of the real ink painting using thinner ink, whose intermission is about one second. According to the intensity of these three strokes, we set the quantity of water to 1.0 and the quantity of carbon to 0.05 to imitate the real ink image. The rendering is shown in Fig. 3a which in the intersection area shown the result of ink-refusal effect as the real ink image in Fig.1a. Fig.3b and Fig.3c show that in the renderings created from the models presented by Kunii[8] and Zhang[12], no any ink-refusal effect appeared.

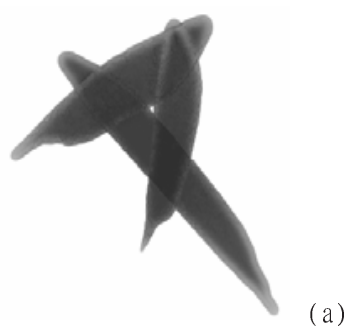

(a)

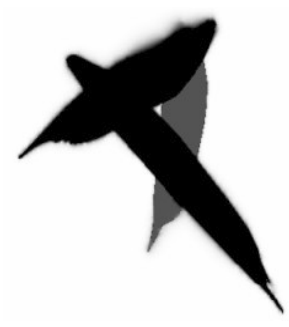

(b)

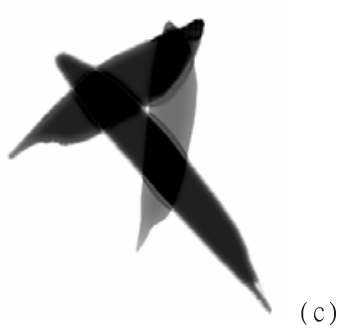

(c)

Fig. 3. The bamboo leaves;(a) our proposed rendering result, (b) Kunii's result, and (c) Zhang's result

We drew a little chicken on the absorbent paper as shown on Fig.1b. Then, we imitate the image by using our model as illustrated in Fig.4a. Compared with both Fig.4b and Fig.4c, which are created by using the models presented by Kunii[8] and Zhang[12], our rendering is more realistic and is able to present the effect of stroketrace-reservation. 


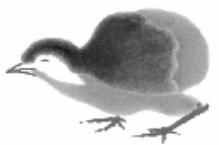

(a)

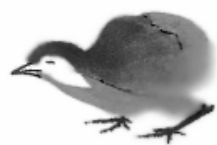

(b)

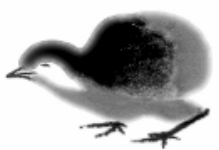

(c)

Fig. 4. A chicken for the effect of stroke-trace-reservation; (a) our proposed rendering result (b) Kunii's result, and (c) Zhang's result

\section{Conclusions}

In this paper we have developed a method that is able to simulate artistic effects such as ink-refusal and stroke-trace-reservation of ink painting. Our method is based on the physical interaction between ink and paper. The main ingredients of ink are water, carbon particles, as well as glue. However, glue is not taken into account in other researches except in ours, although it plays an important role in ink diffusion. We simulate the physical interaction among water, carbon particles, glue, and fiber mesh of paper. The realistic renderings created from our models have demonstrated that our models are successful, and are able to imitate the special artistic effects of ink painting that cannot be rendered from other existing models.

\section{Acknowledgement}

This work was partially supported by National Science Council of Taiwan, R.O.C. under the Grant NSC93-2213-E-029-013.

\section{References}

[1] S. Strassmann, "Hairy brushes," ACM SIGGRAPH (1986) 20(3), 225-232

[2] M.P. Salisbury, S.E. Anderson, R. Barzel, and D.H. Salesin, "Orientable texture for image-based pen-and-ink illustration," 24th annual conference on Computer graphics \& interactive techniques, ACM SIGGRAPH, ACM Press (1997) 401-406

[3] P. Litwinowicz, "Processing images and video for an impressionist effect," Proc. of ACM SIGGRAPH (1997)

[4] K. Perlin, "An image synthesizer," Computer Graphics Proceedings, ACM SIGGRAPH, ACM Press (1985) 199-201

[5] D. Small, "Modeling watercolor by simulating diffusion, pigment, and paper fibers," In Proceedings of SPIE (1991)

[6] C.J. Curtis, S.E. Anderson, J.E. Seims, K.W. Fleischer, and D.H. Salesin, "Computergenerated watercolor," Computer Graphics Proceedings, Annual Conference Series (1997) 421-429

[7] Q. Guo and T.L. Kunii, "Modeling the diffusion painting of sumie," Modeling in Computer Graphics (Proceedings of the IFIP WG5.10), Berlin: (1991) 329-338

[8] T.L. Kunii, G.V. Nosovskij, and H. Takafumi, "A diffusion model for computer animation of diffuse ink painting," Computer Animation ' proceeding (1995) 98-102 
[9] S.Lee, H.Y. Lee, I.F. Lee, and C-Y Tseng, "Ink diffusion in water," European Journal of Physics (2004) 331-336

[10] C.M. Wang, J.S. Lee, and R.J.Wang, "On realistic ink diffusion synthesis for a calligraphic learning system," International Journal of Computer Processing of Oriental Languages (2003) 16(2), 105-118

[11] S.W. Huang, D.L. Way, and Z.C. Shih, "Physical-based model of ink diffusion in Chinese ink paintings," Journal of WSCG'(2003)

[12] Q.Zhang, Y.Sato, J.Takahashi, K.Muraoka, and N.Chiba, "Simple cellular automatonbased simulation of ink behavior and its application to Suibokuga-link 3D rendering of trees," The Journal of Visualization and Computer Animation (1999) 10, 27-37

[13] Q. Guo and T.L. Kunii, "Nijimi rendering algorithm for creating quality black ink painting", Int. conf. on IEEE Computer Graphics-CGI (2003) 